\title{
Self-adjoint Extensions of Schrödinger Operators with $\delta$-magnetic Fields on Riemannian Manifolds
}

\author{
T. Mine
}

\begin{abstract}
We consider the magnetic Schrödinger operator on a Riemannian manifold $M$. We assume the magnetic field is given by the sum of a regular field and the Dirac $\delta$ measures supported on a discrete set $\Gamma$ in $M$. We give a complete characterization of the self-adjoint extensions of the minimal operator, in terms of the boundary conditions. The result is an extension of the former results by Dabrowski-Štovíček and Exner-Štovíček-Vytřas.
\end{abstract}

Keywords: Spectral theory, functional analysis, self-adjointness, Aharonov-Bohm effect, quantum mechanics, differential geometry, Schrödinger operator.

\section{Introduction}

Let $(M, g)$ be a two-dimensional, oriented, connected complete $C^{\infty}$-Riemannian manifold, where $g$ is the Riemannian metric on $M$. Let $d \mu$ be the measure induced from the Riemannian metric. If we take a local chart $(U, \varphi), \varphi=\left(x^{1}, x^{2}\right)$, the measure $d \mu$ is written as $d \mu=\sqrt{G} d x^{1} d x^{2}$ in $U$, where $G=\operatorname{det}\left(g_{m n}\right)$, $g_{m n}=g\left(\partial_{m}, \partial_{n}\right)$, and $\partial_{m}=\partial / \partial x^{m}$. We denote $L^{2}(M)=L^{2}(M ; d \mu)$. The set of all 1-forms on $M$ is denoted by $\Lambda^{1}(M)$. In the coordinate neighbor$\operatorname{hood} U, A \in \Lambda^{1}(M)$ is written as

$$
A=A_{1} d x^{1}+A_{2} d x^{2} .
$$

In general, the coefficients $A_{1}, A_{2}$ are complexvalued. We say $A$ is real-valued if the coefficients are real-valued. We say $A$ is of the class $C^{k} \Lambda^{1}(M)$ if the coefficients are of the class $C^{k}(U)$ for any local chart $(U, \varphi)$. We define the class $L_{\mathrm{loc}}^{q} \Lambda^{1}(M)(1 \leq q \leq \infty)^{1}$, etc. similarly. The 2 -form $d A$ is called the magnetic field. If $A \in L_{\mathrm{loc}}^{1} \Lambda^{1}(M), d A$ can be defined at least in the distribution sense. In $U$, the magnetic field is given by

$$
d A=\left(\partial_{1} A_{2}-\partial_{2} A_{1}\right) d x^{1} \wedge d x^{2} .
$$

Let $\Gamma=\left\{\gamma_{k}\right\}_{k=1}^{K}$ be a sequence of mutually distinct points in $M$. The number $K$ may be infinity, and in this case we assume additionally $\Gamma$ has no accumulation points in $M$. Let $A$ be a 1 -form on $M$ given by the sum of two 1 -forms

(A) $A=A^{(0)}+A^{(1)}$.

The part $A^{(0)}$ corresponds to the $\delta$ magnetic fields, that is, we assume the following.
(A0) $A^{(0)} \in C^{\infty} \Lambda^{1}(M \backslash \Gamma) \cap L_{\text {loc }}^{1} \Lambda^{1}(M)$, real-valued, and

$$
d A^{(0)}=\sum_{k=1}^{K} 2 \pi \alpha_{k} \delta_{\gamma_{k}}
$$

where $\alpha_{k} \in \mathbb{R}$, and $\delta_{\gamma}$ is the Dirac measure concentrated on the point $\gamma$.

More precisely, (1) means

$$
-\int_{M} \mathrm{~d} \varphi \wedge A^{(0)}=\sum_{k=1}^{K} 2 \pi \alpha_{k} \varphi\left(\gamma_{k}\right)
$$

for any $\varphi \in C_{0}^{\infty}(M)$ (since $A^{(0)} \in L_{\text {loc }}^{1} \Lambda^{1}(M)$, the left hand side is well-defined). Notice that this equation is independent of the Riemannian metric $g$. For the regular part $A^{(1)}$ and the scalar potential $V$, we assume the following:

(A1) $A^{(1)} \in C^{1} \Lambda^{1}(M)$, real-valued.

(V) $V$ is real-valued, $V \in L_{\text {loc }}^{2}(M)$, and is bounded in some open neighborhood of $\gamma_{k}$ for every $k=1, \ldots, K$.

Using the local coordinate $\left(x^{1}, x^{2}\right)$, we define the Schrödinger operator $\mathcal{L}$ in each coordinate neighborhood by

$$
\begin{aligned}
\mathcal{L} u= & -\frac{1}{\sqrt{G}} \sum_{m, n=1,2}\left(\partial_{m}+i A_{m}\right) . \\
& \left(\sqrt{G} g^{m n}\left(\partial_{n}+i A_{n}\right) u\right)+V u,
\end{aligned}
$$

\footnotetext{
${ }^{1}$ The measure $d \mu$ is omitted, since the class $L_{\text {loc }}^{q} \Lambda^{1}(M ; d \mu)$ is independent of the choice of $d \mu$. The coefficient $A_{m}$ is a function on $U \subset M$, however, we denote the pull-back $\left(\varphi^{-1}\right)^{*} A_{m}=A_{m} \circ \varphi^{-1}$ on $\varphi(U) \subset \mathbb{R}^{2}$ by the same symbol $A_{m}$, for simplicity of notations. This convention is frequently used in this paper.
} 
where $\left(g^{m n}\right)$ is the inverse matrix of $\left(g_{m n}\right)$. This definition is independent of the choice of local coordinates (see section 2). Define the minimal operator $H_{\text {min }}$ by

$$
H_{\min } u=\mathcal{L} u, \quad D\left(H_{\min }\right)=\overline{C_{0}^{\infty}(M \backslash \Gamma)},
$$

where the overline denotes the closure with respect to the graph norm. Define the maximal operator $H_{\max }$ by $H_{\max }=H_{\min }^{*}$. Then we can show that

$$
\begin{aligned}
H_{\max } u & =\mathcal{L} u, \\
D\left(H_{\max }\right) & =\left\{u \in L^{2}(M) \mid \mathcal{L} u \in L^{2}(M)\right\},
\end{aligned}
$$

where $\mathcal{L}$ is a differential operator on $\mathcal{D}^{\prime}(M \backslash \Gamma)$. We assume

(SB) The operator $H_{\text {min }}$ is bounded from below.

In the case $M$ is the flat Euclidean plane, it is well-known that the operator $H_{\text {min }}$ is not essentially self-adjoint and the structure of the self-adjoint extensions of $H_{\min }$ can be determined via the celebrated Krein-Von Neumann theory of self-adjoint extensions (see e.g. Reed-Simon [13]). In the textbook by Albeverio et al. [3], the case $A^{(0)}=A^{(1)}=0$ and $V=0$ (but $\Gamma \neq \emptyset$ ) is exhaustively studied. AdamiTeta [1] and Dabrowski-Stovíček [7] study the case $K=1, \alpha_{1} \notin \mathbb{Z}, A^{(1)}=0$, and $V=0$. ExnerStovíček-Vytřas [8] study the case $K=1, \alpha_{1} \notin \mathbb{Z}$, $d A^{(1)}=B d x^{1} \wedge d x^{2}$ for some non-zero constant $B$ (the constant magnetic field), and $V=0$. Moreover, Lisovyy [11] studies the case $M$ is the Poincaré disk, $g$ is the Poincaré metric, $V=0$ and $d A=B \omega_{g}+2 \pi \alpha \delta_{0}$, where $B$ is a non-zero constant and $\omega_{g}$ is the surface form induced from the Poincaré metric $g$.

In all the results above, they first determine the deficiency subspaces $\operatorname{Ker}\left(H_{\max } \mp i\right)$ and apply the Krein-Von Neumann theory. This method cannot be applied in the case $K \geq 2$ and $\alpha_{k} \notin \mathbb{Z}$, however, this case (and $A^{(1)}$ is the constant field, $V=0$ ) on the flat Euclidean plane is studied by the author [12], and the structure of the self-adjoint extensions is determined. Our main purpose in this paper is to generalize the result in [12] on general complete Riemannian manifolds and for more general $A$ and $V$.

Our first result is about the deficiency indices $n_{ \pm}\left(H_{\min }\right)=\operatorname{dim} \operatorname{Ker}\left(H_{\max } \mp i\right)$.

Theorem 1.1 Assume (A), (AO), (A1), (V), and $(S B)$. Then, both deficiency indices $n_{ \pm}\left(H_{\min }\right)$ are equal to $2 K_{1}+K_{2}$, where

$$
K_{1}=\#\left\{\alpha_{k} \mid \alpha_{k} \notin \mathbb{Z}\right\}, \quad K_{2}=\#\left\{\alpha_{k} \mid \alpha_{k} \in \mathbb{Z}\right\} .
$$

Note that Bulla-Gesztesy [4] obtain a similar result in the case $A=0$ and $V$ has singularities, and IwaiYabu [9] also obtain a similar result on the twodimensional torus.
Next, we shall give a complete characterization of the self-adjoint extensions of $H_{\mathrm{min}}$. To this purpose, we introduce some nice coordinates around singularities and some auxiliary functions. For simplicity, we assume $K=\# \Gamma$ is finite for a while.

For $k=1, \ldots, K$, let $\left(U_{k}, \phi_{k}\right), \phi_{k}=\left(x^{1}, x^{2}\right)$, be a local chart around $\gamma_{k}$ such that $U_{k}$ is simply connected, $\phi_{k}\left(\gamma_{k}\right)=0, V$ is bounded in $U_{k}$, and $\left\{U_{k}\right\}_{k=1}^{K}$ are disjoint. Let $(r, \theta)$ be the radial coordinate in $U_{k}$ defined by $x^{1}+i x^{2}=r e^{i \theta}, r \geq 0,0 \leq \theta<2 \pi$. We assume

$$
\begin{aligned}
g_{m n}(0,0) & =\delta_{m n}, \\
\partial_{j} g_{m n}(0,0) & =0(m, n, j=1,2),
\end{aligned}
$$

where $\delta_{m n}$ is the Kronecker delta. Condition (2) is satisfied, for example, if we take the normal coordinate ${ }^{2}$ as $\left(x^{1}, x^{2}\right)$.

Let $\beta_{k}$ be the fractional part of $\alpha_{k}$, that is, $\alpha_{k}=\left[\alpha_{k}\right]+\beta_{k},\left[\alpha_{k}\right] \in \mathbb{Z}$ and $0 \leq \beta_{k}<1$. Put $^{3}$

$$
\begin{aligned}
& \tilde{A}^{(0)}=\beta_{k} r^{-2}\left(-x^{2} d x^{1}+x^{1} d x^{2}\right), \\
& \tilde{A}^{(1)}=A^{(1)}-A^{(1)}(0) .
\end{aligned}
$$

It is well-known that $d \tilde{A}^{(0)}=2 \pi \beta_{k} \delta_{0}$ (see e.g. Aharonov-Bohm $[2,1]$ or [7]). Define a phase function $\psi_{k} \in C^{\infty}\left(U_{k} \backslash\{0\}\right)$ by

$$
\begin{aligned}
\psi_{k}(x)= & \exp \frac{1}{i}\left(A_{1}^{(1)}(0) x^{1}+A_{2}^{(1)}(0) x^{2}+\right. \\
& \left.\int_{x_{0}}^{x}\left(A^{(0)}-\tilde{A}^{(0)}\right)\right)
\end{aligned}
$$

where $A^{(1)}=A_{1}^{(1)} d x^{1}+A_{2}^{(1)} d x^{2}, x_{0}$ is some point in $U_{k} \backslash\{0\}$, and the path of the line integral $\int_{x_{0}}^{x}$ lies in $U_{k} \backslash\{0\}$. Notice that the value of the line integral is independent of the choice of paths modulo $2 \pi \mathbb{Z}$, by the Stokes theorem and the assumption $d\left(A^{(0)}-\tilde{A}^{(0)}\right)=2 \pi\left[\alpha_{k}\right] \delta_{0}$ in $U_{k}$. Then we have

$$
A=\tilde{A}+i \psi_{k}^{-1} d \psi_{k}, \quad \tilde{A}=\tilde{A}^{(0)}+\tilde{A}^{(1)}
$$

and

$$
\mathcal{L}=\psi_{k} \tilde{\mathcal{L}} \psi_{k}^{-1}
$$

in $U_{k} \backslash\{0\}$, where $\tilde{\mathcal{L}}$ is the operator $\mathcal{L}$ corresponding to the vector potential $\tilde{A}$ and the scalar potential $V$.

Let $K_{1}, K_{2}$ be the numbers in Theorem 1.1. In the sequel, we rearrange the index $k$ so that $0<\beta_{k}<1$ for $1 \leq k \leq K_{1}$. As we prove later, the

\footnotetext{
${ }^{2}$ The coordinate defined by the local inverse map of the exponential map from the tangent space at $\gamma_{k}$ to $M$.

${ }^{3}$ More precisely, the 1 -form $A^{(1)}-A^{(1)}(0)$ is defined as $\left(A_{1}^{(1)}\left(x^{1}, x^{2}\right)-A_{1}^{(1)}(0,0)\right) d x^{1}+\left(A_{2}^{(1)}\left(x^{1}, x^{2}\right)-A_{2}^{(1)}(0,0)\right) d x^{2}$, in the coordinate neighborhood $U_{k}$.
} 
asymptotics of $u \in D\left(H_{\max }\right)$ in $U_{k}$ as $r \rightarrow 0$ is given by

$$
u= \begin{cases}\psi_{k}\left(c_{1}^{k} r^{\beta_{k}-1} e^{-i \theta}+c_{2}^{k} r^{-\beta_{k}}+\right. & \\ \left.c_{4}^{k} r^{1-\beta_{k}} e^{-i \theta}+c_{5}^{k} r^{\beta_{k}}\right)+\xi & \left(1 \leq k \leq K_{1}\right), \\ \psi_{k}\left(c_{3}^{k} \log r+c_{6}^{k}\right)+\xi \quad\left(K_{1}+1 \leq k \leq K\right),\end{cases}
$$

where $c_{1}^{k}, \ldots, c_{6}^{k}$ are constants and $\xi$ is a regular function in the sense $\xi \in D\left(H_{\min }\right)$. Define

$$
\begin{gathered}
\Phi_{j}(u)= \begin{cases}{ }^{t}\left(c_{j}^{1}, \ldots, c_{j}^{K_{1}}\right) \in \mathbb{C}^{K_{1}} & (j=1,2,4,5), \\
{ }^{t}\left(c_{j}^{K_{1}+1}, \ldots, c_{j}^{K}\right) \in \mathbb{C}^{K_{2}} & (j=3,6),\end{cases} \\
\Phi(u)={ }^{t}\left({ }^{t} \Phi_{1}(u) \cdots{ }^{t} \Phi_{6}(u)\right) \in \mathbb{C}^{4 K_{1}+2 K_{2}} .
\end{gathered}
$$

Define a $\left(2 K_{1}+K_{2}\right) \times\left(2 K_{1}+K_{2}\right)$-diagonal matrix $D$ by

$$
\begin{aligned}
D= & \operatorname{diag}\left(1-\beta_{1}, \ldots, 1-\beta_{K_{1}}, \beta_{1}, \ldots, \beta_{K_{1}},\right. \\
& -1 / 2, \ldots,-1 / 2) .
\end{aligned}
$$

Now our theorem is stated as follows.

Theorem 1.2 Assume (A), (AO), (A1), (V), (SB) and $K<\infty$. Let $\Phi(u), D$ given above.

(i) Let $X=\left(\begin{array}{c}X_{1} \\ X_{2}\end{array}\right)$, where $X_{1}, X_{2}$ are $\left(2 K_{1}+K_{2}\right) \times$ $\left(2 K_{1}+K_{2}\right)$ matrices satisfying

$\operatorname{rank} X=2 K_{1}+K_{2}, X_{1}^{*} D X_{2}=X_{2}^{*} D X_{1}$.

Then, the operator $H_{X}$ defined by

$$
\begin{aligned}
H_{X} u & =\mathcal{L} u, \\
D\left(H_{X}\right) & =\left\{u \in D\left(H_{\max }\right) \mid \Phi(u) \in \operatorname{Ran} X\right\}
\end{aligned}
$$

is a self-adjoint extension of $H_{\min }$.

(ii) For any self-adjoint extension $H$ of $H_{\min }$, there exists some matrix $X$ satisfying (7) and $H=$ $H_{X}$.

We can consider the case $K=\infty$, but some technical assumptions are necessary. We shall argue this case in section 5 .

Thus we can characterize the self-adjoint extensions in terms of the boundary conditions. We can easily prove that the Friedrichs extension corresponds to the case $X_{1}=O, X_{2}=I d$. In the case $M=\mathbb{R}^{2}$ and $K=1$, similar results are obtained in [7] and [8], and our theorem is a generalization of their results. As stated in their paper, the choice of matrices $X$ is of course not unique: there are infinitely many matrices $X$ giving same $\operatorname{Ran} X$.

The difficulty in the proof is that we cannot determine the deficiency subspaces explicitly. To overcome this difficulty, we describe the condition of the self-adjointness only using the quotient subspace
$D\left(H_{\max }\right) / D\left(H_{\min }\right)$. This quotient subspace is essentially the same object as the sum of deficiency subspaces, but much easily tractable than the deficiency subspaces themselves. This idea is also used in [4] or [12].

We note that recently self-adjoint extensions of the Schrödinger operators on $\mathbb{R}^{2}$ with $\delta$ magnetic fields are studied from the viewpoint of the hidden supersymmetric structure; see Correa et al. [5, 6].

The rest of the paper is organized as follows. In section 2, we review basic notations and facts from the differential geometry and the theory of selfadjoint extensions. In section 3, we shall prove the structure of the self-adjoint extensions depends only on the singular part of the vector potentials. In section 4 , we shall prove the main theorems. In section 5 , we shall consider the case $K=\infty$ and give a complete characterization of the self-adjoint extensions, under some homogeneity conditions.

\section{Basic facts}

\subsection{Formulas in differential geometry}

We quote some formulas used in Shubin [14] for the convenience of the readers. Take a local chart $(U, \varphi)$, $\varphi=\left(x^{1}, x^{2}\right)$, around $p \in M$. Put $g_{m n}=g\left(\partial_{m}, \partial_{n}\right)$, and let $\left(g^{m n}\right)$ be the inverse matrix of $\left(g_{m n}\right)$. For $\alpha, \beta \in \Lambda_{p}^{1}(M)$ (the cotangent space at $p$ ), we define the scalar product

$$
\langle\alpha, \beta\rangle=\sum_{m, n=1,2} g^{m n} \alpha_{m} \beta_{n},
$$

where $\alpha=\alpha_{1} d x^{1}+\alpha_{2} d x^{2}$ and $\beta=\beta_{1} d x^{1}+\beta_{2} d x^{2}$. Put $|\alpha|^{2}=\langle\bar{\alpha}, \alpha\rangle$, where $\bar{\alpha}=\overline{\alpha_{1}} d x^{1}+\overline{\alpha_{2}} d x^{2}$. For a 1 -form $\omega=\omega_{1} d x^{2}+\omega_{2} d x^{2}$, we define a function $d^{*} \omega$ by

$$
d^{*} \omega=-\frac{1}{\sqrt{G}} \sum_{m, n=1,2} \partial_{m}\left(\sqrt{G} g^{m n} \omega_{n}\right) .
$$

This definition is independent of the choice of local coordinates. Actually, operator $d^{*}$ is characterized by the following relation:

$$
\int_{M}\langle\overline{d u}, \omega\rangle \mathrm{d} \mu=\int_{M} \bar{u} d^{*} \omega \mathrm{d} \mu
$$

for any $u \in C_{0}^{\infty}(M)$ and $\omega \in C_{0}^{\infty} \Lambda^{1}(M)$.

Let $A$ be a 1 -form satisfying our assumptions. For a function $f$, we define a 1 -form $d_{A} f$ by

$$
d_{A} f=d f+i f A,
$$

where $d$ is the exterior derivative, and $i=\sqrt{-1}$. For a 1 -form $\omega$, we define

$$
d_{A}^{*} \omega=d^{*} \omega-i A^{*} \omega, \quad A^{*} \omega=\langle A, \omega\rangle .
$$


Then we obtain a representation of our Schrödinger operator $\mathcal{L}$ independent of local coordinates:

$$
\mathcal{L}=d_{A}^{*} d_{A}+V .
$$

For operator $d_{A}^{*}$, the following Leibniz formulas hold: for an appropriate function $f$ and 1-form $\omega$, we have

$$
\begin{aligned}
d_{A}^{*}(f \omega)= & f d^{*} \omega-\langle d f, \omega\rangle-i f\langle A, \omega\rangle= \\
& f d_{A}^{*} \omega-\langle d f, \omega\rangle=f d^{*} \omega-\left\langle d_{A} f, \omega\right\rangle, \\
d_{A}^{*} d_{A}(f g)= & f d_{A}^{*} d_{A} g-2\left\langle d f, d_{A} g\right\rangle+g d^{*} d f .
\end{aligned}
$$

Proposition 2.1 Let $U, U^{\prime}$ be open subsets of $M \backslash \Gamma$ such that $\bar{U}$ is a compact subset of $U^{\prime}$, and $V$ is bounded in $U^{\prime}$. Then, there exists a constant $C>0$ such that

$$
\int_{U}\left|d_{A} f\right|^{2} \mathrm{~d} \mu \leq C \int_{U^{\prime}}\left(|f|^{2}+|\mathcal{L} f|^{2}\right) \mathrm{d} \mu
$$

for $f \in D\left(H_{\max }\right)$.

Proof. According to $[14,(5.3)],{ }^{4}$ we have

$$
(\mathcal{L}(\phi f), \phi f)=\Re(\phi \mathcal{L} f, \phi f)+\int_{M}|d \phi|^{2}|f|^{2} \mathrm{~d} \mu
$$

for $f \in D\left(H_{\max }\right)$ and $\phi \in C_{0}^{\infty}(M \backslash \Gamma)$. Take $\phi \in C_{0}^{\infty}\left(U^{\prime}\right)$ such that $\phi=1$ on $U$. Then the conclusion follows from the above equality,

$$
\int_{\operatorname{supp} \phi}\left|d_{A}(\phi f)\right|^{2}=(\mathcal{L}(\phi f), \phi f)-(V \phi f, \phi f)
$$

and assumption $V$ is bounded.

\subsection{Theory of self-adjoint extensions}

We quote some notation from the textbook [13]. Let $\mathcal{H}$ be a separable Hilbert space and denote its inner product by $(\cdot, \cdot)$, and norm by $\|\cdot\|$. All the linear operators in this subsection are on the Hilbert space $\mathcal{H}$. For a linear operator $X, D(X)$ denotes the domain of definition of $X, \bar{X}$ the closure of $X, X^{*}$ the adjoint operator of $X$. For a linear operator $X$, the graph inner product of $X$ is defined by

$$
(x, y)_{X}=(X x, X y)+(x, y)
$$

for $x, y \in D(X)$, and the graph norm by $\|x\|_{X}=$ $(x, x)_{X}^{1 / 2}$.

We introduce some equivalent for the sum of the deficiency subspaces, which is also introduced in [4] or [12]. Let $X$ be a closed, densely defined symmetric operator. Let $\mathcal{D}=D\left(X^{*}\right) / D(X)$, where the right hand side denotes the quotient space. The space $\mathcal{D}$ is a Hilbert space equipped with the norm

$$
\|[x]\|_{\mathcal{D}}^{2}=\min _{y \in[x]}\|y\|_{X^{*}}^{2}=\|Q x\|_{X^{*}}^{2}
$$

where $x \in D\left(X^{*}\right),[x]=x+D(X)$ denotes the equivalence class of $x$ in the quotient space $D\left(X^{*}\right) / D(X)$, and $Q$ denotes the orthogonal projection onto the orthogonal complement of $D(X)$ in $D\left(X^{*}\right)$. For $u, v \in \mathcal{D}$, define

$$
\begin{aligned}
{[u, v]_{\mathcal{D}} } & =\left(X^{*} x, y\right)-\left(x, X^{*} y\right) \\
u & =[x], v=[y], x, y \in D\left(X^{*}\right)
\end{aligned}
$$

The value $[u, v]_{\mathcal{D}}$ is independent of the choice of the representatives $x, y$. Let $P$ be the canonical projection from $D\left(X^{*}\right)$ to $\mathcal{D}$. For a closed subspace $V$ of $\mathcal{D}$, we define a closed linear operator $X_{V}$ by

$$
D\left(X_{V}\right)=\left\{x \in D\left(X^{*}\right) \mid P x \in V\right\}, \quad X_{V} x=X^{*} x .
$$

We also define

$$
V^{[\perp]}=\left\{u \in \mathcal{D} \mid[u, v]_{\mathcal{D}}=0 \text { for any } v \in V\right\} .
$$

Then the following proposition immediately follows from the definition of the self-adjointness.

Proposition 2.2 1. For a closed subspace $V$ of $\mathcal{D}$, the operator $X_{V}$ is a self-adjoint extension of $X$ if and only if

$$
V^{[\perp]}=V .
$$

2. For any self-adjoint extension $\tilde{X}$ of $X$, there exists a closed subspace $V$ of $\mathcal{D}$ such that $X_{V}=\tilde{X}$.

In terms of the above notations, the Krein-Von Neumann theory can be rephrased as follows.

Proposition 2.3 Let $\mathcal{N}_{ \pm}=\operatorname{Ker}\left(X^{*} \mp i\right)$ the deficiency subspaces of $X, n_{ \pm}=\operatorname{dim} \mathcal{N}_{ \pm}$the deficiency indices of $X$. Then, the following holds.

(i) The projection operator $P$ gives a Hilbert space isomorphism from the direct sum $\mathcal{N}_{+} \oplus \mathcal{N}_{-}$to $\mathcal{D}$. In particular, $\operatorname{dim} \mathcal{D}=n_{+}+n_{-}$.

(ii) There exists a one-to-one correspondence between the closed subspaces $V$ of $\mathcal{D}$ satisfying (11) and the unitary operators $U$ from $\mathcal{H}_{+}$to $\mathcal{H}_{-}$, given by

$$
V=P(1+U) \mathcal{H}_{+} .
$$

This proposition says the space $\mathcal{D}$ can play the same role as the sum of deficiency subspaces in the theory of self-adjoint extensions. Particularly when $\mathcal{N}_{ \pm}$ is difficult to determine explicitly (as in our case), the space $\mathcal{D}$ is more tractable, since the element of this space has ambiguity by $D(X)$. Actually, in the next section we shall see that the structure of $\mathcal{D}$ for our Schrödinger operator $H_{\min }$ and the form $[\cdot, \cdot]_{\mathcal{D}}$ is determined only from the singular part $A^{(0)}$ of the vector potential.

\footnotetext{
${ }^{4}$ Since the function $\phi$ avoids the singularities, the proof of $[14,(5.3)]$ is also available in our case.
} 


\section{Reduction}

\subsection{Division to the local potential}

Let $\left(U_{k}, \phi_{k}\right), \phi_{k}=\left(x^{1}, x^{2}\right)$, the local coordinate introduced in section 1 . Let $\tilde{A}$ be the 1 -form given by (4). Take a positive number $\epsilon_{k}$ so small that the closed disc $\left\{r \leq 2 \epsilon_{k}\right\}$ is contained in $U_{k}$. Let $\eta_{k} \in C_{0}^{\infty}(U)$ such that $0 \leq \eta_{k} \leq 1, \eta_{k}=1$ for $r \leq \epsilon_{k}$, $\eta_{k}=0$ for $r \geq 2 \epsilon_{k}$. Define functions $\hat{g}_{m n}, \hat{A}_{m}$ and $\hat{V}$ on $\mathbb{R}^{2}$ by

$$
\begin{aligned}
\hat{g}_{m n} & =\eta_{k} g_{m n}+\left(1-\eta_{k}\right) \delta_{m n} \\
\hat{A}_{m} & =\tilde{A}_{m}^{(0)}+\eta_{k} \tilde{A}_{m}^{(1)} \\
\hat{V} & =\eta_{k} V .
\end{aligned}
$$

Define a differential operator $\mathcal{L}_{k}$ on $\mathbb{R}^{2}$ by

$$
\begin{aligned}
& \mathcal{L}_{k}=-\frac{1}{\sqrt{\hat{G}}} \sum_{m, n=1,2}\left(\frac{\partial}{\partial x_{m}}+i \hat{A}_{m}\right) . \\
& \sqrt{\hat{G}} \hat{g}^{m n}\left(\frac{\partial}{\partial x_{n}}+i \hat{A}_{n}\right)+\hat{V}
\end{aligned}
$$

where $\hat{G}=\operatorname{det}\left(\hat{g}_{m n}\right)$, and $\left(\hat{g}^{m n}\right)$ is the inverse matrix of $\left(\hat{g}_{m n}\right)$. Define a linear operator $L_{k, \min }$ on $L^{2}\left(\mathbb{R}^{2} ; d \mu_{k}\right), d \mu_{k}=\sqrt{\hat{G}} d x^{1} d x^{2}$, by

$$
L_{k, \min } u=\mathcal{L}_{k} u, \quad D\left(L_{k, \min }\right)=\overline{C_{0}^{\infty}\left(\mathbb{R}^{2} \backslash\{0\}\right)} .
$$

Let $L_{k, \max }=L_{k, \min }^{*}$. Then

$$
L_{k, \max } u=\mathcal{L}_{k} u,
$$

$D\left(L_{k, \text { max }}\right)=\left\{u \in L^{2}\left(\mathbb{R}^{2} ; d \mu_{k}\right) \mid \mathcal{L}_{k} u \in L^{2}\left(\mathbb{R}^{2} ; d \mu_{k}\right)\right\}$,

where $\mathcal{L}_{k}$ is regarded as a differential operator on $\mathcal{D}^{\prime}\left(\mathbb{R}^{2} \backslash 0\right)$. Let $\mathcal{D}=D\left(H_{\max }\right) / D\left(H_{\min }\right), \mathcal{D}_{k}=$ $D\left(L_{k, \max }\right) / D\left(L_{k, \min }\right)$. Let $\chi_{k} \in C_{0}^{\infty}(M)$ such that $0 \leq \chi_{k} \leq 1, \chi_{k}=0$ for $r \geq \epsilon_{k}$ and $\chi_{k}=1$ for $r \leq \epsilon_{k} / 2$. Define a map $T_{k}$ from $\mathcal{D}$ to $\mathcal{D}_{k}$ by

$$
T_{k}[f]=\left[\psi_{k}^{-1} \chi_{k} f\right],
$$

where the function $\psi_{k}$ is given by (3). Define a map $T$ from $\mathcal{D}$ to the direct sum $\bigoplus_{k=1}^{K} \mathcal{D}_{k}$ by

$$
T[f]=\bigoplus_{k=1}^{k} T_{k}[f]
$$

We also define a map $S$ from $\bigoplus_{k=1}^{K} \mathcal{D}_{k}$ to $\mathcal{D}$ by $^{5}$

$$
S \bigoplus_{k=1}^{K}\left[f_{k}\right]=\left[\sum_{k=1}^{K} \psi_{k} \chi_{k} f_{k}\right]
$$

In the sequel, we sometimes write $[f, g]_{\mathcal{D}}=\left[[f],[g]_{\mathcal{D}}\right.$ etc. for simplicity of notations.

Lemma 3.1 1. Assume $K<\infty$. Then, the maps $S, T$ defined above are well-defined and mutually inverse. Moreover, we have

$$
[f, g]_{\mathcal{D}}=\sum_{k=1}^{k}\left[T_{k}[f], T_{k}[g]\right]_{\mathcal{D}_{k}}
$$

for any $[f],[g] \in \mathcal{D}$.

2. Assume $K=\infty$. Then the map $S$ is well-defined and injective.

Proof. (i) We divide the proof into three steps.

Step 1. The map

$$
D\left(H_{\max }\right) \ni f \mapsto \psi_{k}^{-1} \chi_{k} f \in D\left(L_{k, \max }\right)
$$

is well-defined and continuous.

Proof. Clearly $\psi_{k}^{-1} \chi_{k} f \in L^{2}\left(\mathbb{R}^{2} ; d \mu_{k}\right)$, so it suffices to show that $\mathcal{L}_{k}\left(\psi_{k}^{-1} \chi_{k} f\right) \in L^{2}\left(\mathbb{R}^{2} ; d \mu_{k}\right)$. By (5) and the Leibniz rule $(9)$, we have

$$
\begin{aligned}
& \mathcal{L}_{k}\left(\psi_{k}^{-1} \chi_{k} f\right)=\psi_{k}^{-1} \mathcal{L}\left(\chi_{k} f\right)= \\
& \psi_{k}^{-1}\left(\chi_{k} \mathcal{L} f-2\left\langle d \chi_{k}, d_{A} f\right\rangle+\left(d^{*} d \chi_{k}\right) f\right) .
\end{aligned}
$$

The first term and the third in the parenthesis of the right hand side are in $L^{2}\left(\mathbb{R}^{2} ; d \mu_{k}\right)$ and continuous with respect to $\|\cdot\|_{H_{\max }}$. Moreover, we can prove the second term is also in $L^{2}$ and continuous with respect to $\|\cdot\|_{H_{\max }}$ by using (10).

Step 2. Let $f \in D\left(H_{\text {min }}\right)$. Then, we have $\psi_{k}^{-1} \chi_{k} f \in$ $D\left(L_{k, \min }\right)$.

Proof. By definition, there exists a sequence $\left\{f_{n}\right\}_{n=1}^{\infty} \subset C_{0}^{\infty}(M \backslash \Gamma)$ such that $f_{n} \rightarrow f$ in $D\left(H_{\text {min }}\right)$. Then, $\psi_{k}^{-1} \chi_{k} f_{n} \in C_{0}^{\infty}\left(\mathbb{R}^{2} \backslash\{0\}\right)$ and $\psi_{k}^{-1} \chi_{k} f_{n} \rightarrow$ $\psi_{k}^{-1} \chi_{k} f$ in $D\left(L_{k, \max }\right)$, by Step 1 . Since $D\left(L_{k, \min }\right)$ is a closed subspace of $D\left(L_{k, \max }\right)$, we have the conclusion.

Step 1 and 2 imply the map $T$ is well-defined. We can similarly prove that the map $S$ is also welldefined.

Step 3. The operator $S T$ is the identity map on $\mathcal{D}$. Proof. By definition, we have

$$
(I-S T)[f]=[\psi f], \quad \psi=1-\sum_{k=1}^{K} \chi_{k}^{2}
$$

So it suffices to prove that $g=\psi f \in D\left(H_{\min }\right)$.

\footnotetext{
${ }^{5}$ When $K=\infty$, we define the map $S$ for the elements of difficulty in the definition of $S$.
} 
Let $(r, \theta)$ be the radial coordinate in $U_{k}$ and put $B_{k, \epsilon}=\left\{x \in U_{k} \mid r<\epsilon\right\}$. Then we have $\operatorname{supp} \psi \subset M \backslash \bigcup_{k=1}^{K} B_{k, \epsilon_{k} / 2}$. For $c>0$, let $\xi_{c} \in C^{\infty}(M)$ such that $0 \leq \xi_{c} \leq 1, \xi_{c}=1$ in $M \backslash \bigcup_{k=1}^{K} B_{\epsilon_{k} / c}, \xi_{c}=0$ in $\bigcup_{k=1}^{K} B_{k, \epsilon_{k} /(2 c)}$. Let $\mathcal{L}_{0}, H_{0, \min }$ and $H_{0, \max }$ be the operators corresponding to the potentials $\xi_{4} A$ and $\xi_{4} V$. These potentials have no singularities, so we have $H_{0, \text { min }}=H_{0, \text { max }}$ by [14]. Since $\mathcal{L} g=\mathcal{L}_{0} g \in L^{2}$, we have $g \in D\left(H_{0, \max }\right)=D\left(H_{0, \min }\right)$. Thus we can take a sequence $\left\{g_{n}\right\}$ such that $g_{n} \rightarrow g$ in $\|\cdot\|_{H_{0, \min }}$. Then $\xi_{2} g_{n} \in C_{0}^{\infty}(M \backslash \Gamma)$ and $\xi_{2} g_{n} \rightarrow \xi_{2} g=g$ in $\|\cdot\|_{H_{\min }}$. Thus we have $g \in D\left(H_{\min }\right)$.

We can prove $T S=I$ similarly. Then (12) follows from (5) and the equality $[f, g]_{\mathcal{D}}=\sum_{k=1}^{K}\left[\chi_{k} f, \chi_{k} g\right]_{\mathcal{D}}$ (notice that $f-\sum_{k} \chi_{k} f \in D\left(H_{\min }\right)$ can be proved as in Step 3).

(2) Let $K=\infty$. For any positive integer $n$, we can define $T^{(n)}$ from $\mathcal{D}$ to $\bigoplus_{k=1}^{n} \mathcal{D}_{k}$, and $S^{(n)}$ from $\bigoplus_{k=1}^{n} \mathcal{D}_{k}$ to $\mathcal{D}$ similarly, and prove $T^{(n)} S^{(n)}=I d$. This implies the map $S$ is well-defined and injective.

\subsection{Analysis of operators on $\mathbb{R}^{2}$}

We shall analyze the operator $\mathcal{L}_{k}$ (or $L_{k, \min }, L_{k, \max }$ ) defined in the previous subsection. For simplicity of notation, we omit ${ }^{\wedge}$ and $\sim$ in the definition of $\mathcal{L}_{k}$ in the sequel. Then our assumptions are the following:

1. $\mathcal{L}_{k}=d_{A}^{*} d_{A}+V$ on $\mathbb{R}^{2} \backslash\{0\}, A=A^{(0)}+A^{(1)}$,

2. $L_{k, \text { min }}$ and $L_{k, \max }$ are operators on $L^{2}\left(\mathbb{R}^{2} ; d \mu_{k}\right)$, $d \mu_{k}=\sqrt{G} d x^{1} d x^{2}$

3. $A^{(0)}=\beta_{k} r^{-2}\left(-x^{2} d x^{1}+x^{1} d x^{2}\right), 0 \leq \beta_{k}<1$,

4. $A^{(1)} \in C_{0}^{1} \Lambda^{1}\left(\left\{r<2 \epsilon_{k}\right\}\right)$, real-valued, $A^{(1)}(0)=$ 0 ,

5. $V$ is bounded, real-valued,

6. $g_{m n}(0)=\delta_{m n}, \partial_{j} g_{m n}(0)=0$, and $g_{m n}=\delta_{m n}$ for $r \geq 2 \epsilon_{k}$.

We shall show that $g_{m n}, A^{(1)}$ and $V$ have nothing to do with the structure of the self-adjoint extensions. To this purpose, define a differential operator $\mathcal{M}_{k}$ on $\mathbb{R}^{2}$ by

$$
\mathcal{M}_{k}=-\sum_{n=1,2}\left(\frac{\partial}{\partial x_{n}}+i A_{n}\right)^{2}
$$

Define a linear operator $M_{k, \text { min }}$ on $L^{2}\left(\mathbb{R}^{2} ; d x^{1} d x^{2}\right)$ by

$$
\begin{aligned}
D\left(M_{k, \min }\right) & =\overline{C_{0}^{\infty}\left(\mathbb{R}^{2} \backslash\{0\}\right)}, \\
M_{k, \min } u & =\mathcal{M}_{k} u \text { for } u \in D\left(M_{k, \min }\right) .
\end{aligned}
$$

Put $M_{k, \text { max }}=M_{k, \text { min }}^{*}$, and $\mathcal{E}_{k}=D\left(M_{k, \max }\right) /$ $D\left(M_{k, \text { min }}\right)$. We also define $\mathcal{M}_{k}^{(0)}, M_{k, \text { min }}^{(0)}, M_{k, \max }^{(0)}$, and $\mathcal{E}_{k}^{(0)}$, by replacing $A_{n}$ by $A_{n}^{(0)}$ in the above definition.

The operator $\mathcal{M}_{k}^{(0)}$ is already studied in [1] and [7]. Here we quote their results and calculate the form $[\cdot, \cdot]_{\mathcal{E}_{k}^{(0)}}$.

Proposition 3.2 Let $\chi \in C_{0}^{\infty}\left(\mathbb{R}^{2}\right)$ such that $\chi=1$ in some neighborhood of 0 .

1. Assume $0<\beta_{k}<1$. Put

$$
\begin{aligned}
& f_{k}^{1}=\chi e^{-i \theta} r^{\beta_{k}-1}, \quad f_{k}^{2}=\chi r^{-\beta_{k}}, \\
& f_{k}^{4}=\chi e^{-i \theta} r^{1-\beta_{k}}, \quad f_{k}^{5}=\chi r^{\beta_{k}} .
\end{aligned}
$$

Then, the deficiency indices $n_{ \pm}\left(M_{k, \min }^{(0)}\right)=2$, $\operatorname{dim} \mathcal{E}_{k}^{(0)}=4$ and the vectors $\left\{\left[f_{k}^{n}\right]\right\}_{n=1,2,4,5}$ form a basis of $\mathcal{E}_{k}^{(0)}$. Moreover, for $m, n \in\{1,2,4,5\}$ with $m \leq n,{ }^{6}$ we have

$$
\left[f_{k}^{m}, f_{k}^{n}\right]_{\mathcal{E}_{k}^{(0)}}= \begin{cases}4 \pi\left(\beta_{k}-1\right) & \text { for }(m, n)=(1,4), \\ -4 \pi \beta_{k} & \text { for }(m, n)=(2,5), \\ 0 & \text { otherwise. }\end{cases}
$$

2. Assume $\beta_{k}=0$. Put

$$
f_{k}^{3}=\chi \log r, \quad f_{k}^{6}=\chi .
$$

Then, the deficiency indices $n_{ \pm}\left(M_{k, \min }^{(0)}\right)=1$, $\operatorname{dim} \mathcal{E}_{k}^{(0)}=2,\left\{\left[f_{k}^{j}\right]\right\}_{j=3,6}$ form a basis of $\mathcal{E}_{k}^{(0)}$, and

$$
\left[f_{k}^{3}, f_{k}^{6}\right]_{\mathcal{E}_{k}^{(0)}}=2 \pi, \quad\left[f_{k}^{3}, f_{k}^{3}\right]_{\mathcal{E}_{k}^{(0)}}=\left[f_{k}^{6}, f_{k}^{6}\right]_{\mathcal{E}_{k}^{(0)}}=0 .
$$

Proof. (i) The first statement follows from the result in [7] or [1]. For the calculation of $[u, v]_{\mathcal{E}_{k}^{(0)}}$, we use some notation in vector analysis. We use the gradient vector $\nabla={ }^{t}\left(\partial_{1}, \partial_{2}\right)$, and identify a 1 -form $A$ with the component vector ${ }^{t}\left(A_{1}, A_{2}\right)$. The dot . denotes the Euclidean inner product. Then we have

$$
\begin{aligned}
{[u, v]_{\mathcal{E}_{k}^{(0)}}=} & \lim _{\epsilon \rightarrow 0} \int_{r \geq \epsilon}\left(-v \overline{\left(\nabla+i A^{(0)}\right) \cdot\left(\nabla+i A^{(0)}\right) u}+\right. \\
& \left.\bar{u}\left(\nabla+i A^{(0)}\right) \cdot\left(\nabla+i A^{(0)}\right) v\right) d x^{1} d x^{2}=
\end{aligned}
$$

\footnotetext{
${ }^{6}$ Notice that $\left[f_{k}^{n}, f_{k}^{m}\right]_{\mathcal{E}_{k}^{(0)}}=-\overline{\left[f_{k}^{m}, f_{k}^{n}\right]_{\mathcal{E}_{k}^{(0)}}}$ by definition.
} 


$$
\begin{aligned}
& \lim _{\epsilon \rightarrow 0} \int_{r=\epsilon}\left(v n \cdot \overline{\left(\nabla+i A^{(0)}\right) u}-\right. \\
& \left.\bar{u} n \cdot\left(\nabla+i A^{(0)}\right) v\right) r \mathrm{~d} \theta= \\
& \lim _{\epsilon \rightarrow 0} \int_{r=\epsilon}\left(v \overline{\partial_{r} u}-\bar{u} \partial_{r} v\right) r \mathrm{~d} \theta,
\end{aligned}
$$

where $n=(\cos \theta, \sin \theta)$, and the line integral is taken counterclockwise. We used the Green formula and the fact $n \cdot A^{(0)}=0$. Then we can easily prove the second statement by using (13).

(ii) The first part of the statement follows from the results in [3]. The second statement can be justified by using (13).

Next, we prove that the regular part $A^{(1)}$ does not affect the structure of $\mathcal{E}_{k}$ and the corresponding form.

Proposition 3.3 All the statements of Proposition 3.2 hold even if we replace $M_{k, \min }^{(0)}$ by $M_{k, \min }$, and $\mathcal{E}_{k}^{(0)}$ by $\mathcal{E}_{k}$.

Before the proof, we prepare a perturbative lemma, which is an immediate corollary of $[10$, Theorem IV.5.22].

Lemma 3.4 Let $\mathcal{H}$ be a separable Hilbert space and $\|\cdot\|$ its norm. Let $X, Y$ be densely defined symmetric operators on $\mathcal{H}$. Assume $D(X) \subset D(Y)$ and there exist positive constants $C, \delta$ with $0<\delta<1$ and

$$
\|Y u\| \leq \delta\|X u\|+C\|u\|
$$

for every $u \in D(X)$. Then, we have $D(\overline{X+Y})=$ $D(\bar{X})$ and $n_{ \pm}(X+Y)=n_{ \pm}(X)$, where the overline denotes the operator closure.

Proof of Proposition 3.3 We prove only statement (i). Statement (ii) can be proved similarly.

By the Leibniz formula (8), we have for $u \in$ $C_{0}^{\infty}\left(\mathbb{R}^{2} \backslash\{0\}\right)$

$$
\begin{aligned}
\left(\mathcal{M}_{k}-\mathcal{M}_{k}^{(0)}\right) u= & i\left(d^{*} A^{(1)}\right) u- \\
& 2 i\left\langle A^{(1)}, d_{A^{(0)}} u\right\rangle+\left|A^{(1)}\right|^{2} u .
\end{aligned}
$$

We denote $\|u\|^{2}=\int_{\mathbb{R}^{2}}|u|^{2} d x^{1} d x^{2}$ for a function $u$, and $\|\omega\|^{2}=\int_{\mathbb{R}^{2}}|\omega|^{2} d x^{1} d x^{2}$ for a 1 -form $\omega$ (notice that $\left.|\omega|^{2}=\langle\bar{\omega}, \omega\rangle\right)$. We denote the essential supremum norm of $|u|$ and $|\omega|$ by $\|u\|_{\infty}$ and $\|\omega\|_{\infty}$, respectively. Then we have by the Schwarz inequality

$\left\|\left(\mathcal{M}_{k}-\mathcal{M}_{k}^{(0)}\right) u\right\| \leq$

$\left\|d^{*} A^{(1)}\right\|_{\infty}\|u\|+2\left\|A^{(1)}\right\|_{\infty}\left\|d_{A^{(0)}} u\right\|+\left\|A^{(1)}\right\|_{\infty}^{2}\|u\| \leq$

$\left(\left\|d^{*} A^{(1)}\right\|_{\infty}+\left\|A^{(1)}\right\|_{\infty}^{2}\right)\|u\|+$

$\left\|A^{(1)}\right\|_{\infty}\left(\epsilon\left\|\mathcal{M}_{k}^{(0)} u\right\|^{2}+\epsilon^{-1}\|u\|^{2}\right)$

for any $\epsilon>0$, where we used the inequality

$$
\left\|d_{A^{(0)}} u\right\|=\left(\mathcal{M}_{k}^{(0)} u, u\right)^{1 / 2} \leq
$$

$$
\begin{aligned}
& \left(\epsilon\left\|\mathcal{M}_{k}^{(0)} u\right\|\right)^{1 / 2}\left(\epsilon^{-1}\|u\|\right)^{1 / 2} \leq \\
& \frac{1}{2}\left(\epsilon\left\|\mathcal{M}_{k}^{(0)} u\right\|+\epsilon^{-1}\|u\|\right) .
\end{aligned}
$$

Take $\epsilon>0$ sufficiently small and apply Lemma 3.4. Then we have $n_{ \pm}\left(M_{k, \text { min }}\right)=n_{ \pm}\left(M_{k, \text { min }}^{(0)}\right)=2$, thus $\operatorname{dim} \mathcal{E}_{k}=4$ by (i) of Proposition 2.3. Moreover we have $D\left(M_{k, \min }\right)=D\left(M_{k, \min }^{(0)}\right)$, so the functions $\left\{f_{k}^{j}\right\}$ $(j=1,2,4,5)$ do not belong to $D\left(M_{k, \min }\right)$. And we can prove $\mathcal{M}_{k} f_{k}^{j} \in L^{2}\left(\mathbb{R}^{2}\right)$ by using (14) and the fact $\left|A^{(1)}\right|=O(r)$ near the origin. Thus $\left\{\left[f_{k}^{j}\right]\right\}$ form a basis of $\mathcal{E}_{k}$. For the form $[\cdot, \cdot]_{\mathcal{E}_{k}}$, we can prove the formula

$$
\begin{aligned}
& {[u, v]_{\mathcal{E}_{k}}=} \\
& \lim _{\epsilon \rightarrow 0} \int_{r=\epsilon}\left(v \overline{\left(\partial_{r} u\right)}-\bar{u}\left(\partial_{r} v\right)-2 i\left(n \cdot A^{(1)}\right) \bar{u} v\right) r d \theta
\end{aligned}
$$

in a similar way as in (13). Thus the value $\left[f_{k}^{m}, f_{k}^{n}\right]_{\mathcal{E}_{k}}$ is not affected by $A^{(1)}$, since $\left|A^{(1)}\right|=O(r)$ and $\left|f_{k}^{m} f_{k}^{n}\right|$ is at most $O\left(r^{-\max \left(2 \beta_{k}, 2\left(1-\beta_{k}\right)\right)}\right)$.

Next we shall consider the non-flat case. We shall show that metric $g$ also does not affect the structure of $\mathcal{D}_{k}$ and the corresponding form.

Proposition 3.5 All the statements of Proposition 3.2 hold even if we replace $M_{k, \min }^{(0)}$ by $L_{k, \min }$ and $\mathcal{E}_{k}^{(0)}$ by $\mathcal{D}_{k}$.

Since $V$ is bounded, we can assume $V=0$. In the sequel, we use the following notation:

$$
\mathcal{L}=G^{-1 / 2}(D+A) \cdot G^{1 / 2} g^{-1}(D+A),
$$

where $D$ is the column vector ${ }^{t}\left(D_{1}, D_{2}\right), D_{j}=-i \partial_{j}$, $A$ is identified with the component vector ${ }^{t}\left(A_{1}, A_{2}\right)$, and $g^{-1}$ is the inverse matrix of $g=\left(g_{m n}\right)$.

We shall prepare some elliptic a priori estimate.

Lemma 3.6 Let $m, n \in\{1,2\}$. Then, there exist $C_{m}>0$ and $C_{m n}>0$ such that

$\left\|\left(D_{m}+A_{m}\right) u\right\| \leq C_{m}\left(\epsilon\left\|\mathcal{M}_{k} u\right\|+\epsilon^{-1}\|u\|\right)$,

$\left\|\left(D_{m}+A_{m}\right)\left(D_{n}+A_{n}\right) u\right\| \leq C_{m n}\left(\left\|\mathcal{M}_{k} u\right\|+\|u\|\right)$

for every $u \in C_{0}^{\infty}\left(\mathbb{R}^{2} \backslash\{0\}\right)$ and every $\epsilon>0$, where $\|\cdot\|=\|\cdot\|_{L^{2}\left(\mathbb{R}^{2} ; d x^{1} d x^{2}\right)}$.

The difficulty is the singularity of our vector potential $A$ at the origin. We can overcome this difficulty by using some commutator technique.

Proof of Lemma 3.6 Put $\Pi_{j}=D_{j}+A_{j}(j=1,2)$. Then, since

$$
\begin{aligned}
\left\|\Pi_{j} u\right\|^{2}= & \left(\epsilon^{1 / 2} \Pi_{j}^{2} u, \epsilon^{-1 / 2} u\right) \leq \\
& \frac{1}{2}\left(\epsilon\left\|\Pi_{j}^{2} u\right\|^{2}+\epsilon^{-1}\|u\|^{2}\right)
\end{aligned}
$$

for $u \in C_{0}^{\infty}\left(\mathbb{R}^{2}\right)$, it suffices to prove (15). 
Define auxiliary operators

$$
\mathcal{A}=i \Pi_{1}+\Pi_{2}, \quad \mathcal{A}^{\dagger}=-i \Pi_{1}+\Pi_{2} .
$$

Let $[X, Y]=X Y-Y X$ be the commutator of operators $X$ and $Y$. Then we have

$$
\left[\Pi_{1}, \Pi_{2}\right]=\left[D_{1}, A_{2}\right]-\left[D_{2}, A_{1}\right]=-i\left(b+2 \pi \beta_{k} \delta_{0}\right),
$$

where $b=\partial_{1} A_{2}^{(1)}-\partial_{2} A_{1}^{(1)}$ is the magnetic field corresponding to $A^{(1)}$. Thus we have

$$
\left[\mathcal{A}, \mathcal{A}^{\dagger}\right]=2 i\left[\Pi_{1}, \Pi_{2}\right]=2\left(b+2 \pi \beta_{k} \delta_{0}\right) .
$$

Particularly for $u \in C_{0}^{\infty}\left(\mathbb{R}^{2} \backslash\{0\}\right)$, we have

$$
\left(\mathcal{A} \mathcal{A}^{\dagger}-\mathcal{A}^{\dagger} \mathcal{A}\right) u=2 b u
$$

Moreover, we have by definition

$$
\left(\mathcal{A} \mathcal{A}^{\dagger}+\mathcal{A}^{\dagger} \mathcal{A}\right) u=2 \mathcal{M}_{k} u
$$

These equalities imply

$$
\mathcal{A} \mathcal{A}^{\dagger}=\mathcal{M}_{k}+b, \quad \mathcal{A}^{\dagger} \mathcal{A}=\mathcal{M}_{k}-b
$$

on $C_{0}^{\infty}\left(\mathbb{R}^{2} \backslash\{0\}\right)$.

Since $\Pi_{m} \Pi_{n}$ can be written as a finite linear combination of the operators of the form $X Y$, where $X, Y$ are $\mathcal{A}$ or $\mathcal{A}^{\dagger}$, it suffices to show that there exists some constant $C>0$ such that

$$
\|X Y u\| \leq C\left(\left\|\mathcal{M}_{k} u\right\|+\|u\|\right)
$$

for $u \in C_{0}^{\infty}\left(\mathbb{R}^{2} \backslash\{0\}\right)$. For $(X, Y)=\left(\mathcal{A}, \mathcal{A}^{\dagger}\right),\left(\mathcal{A}^{\dagger}, \mathcal{A}\right)$, (17) follows from (16), since $b$ is bounded. To estimate $\left\|\mathcal{A}^{2} u\right\|^{2}$, we assume $A^{(1)} \in C^{\infty}$ for a while. Then, we have by (16)

$$
\begin{aligned}
& \left\|\mathcal{A}^{2} u\right\|^{2}=\left(\mathcal{A}^{2} u, \mathcal{A}^{2} u\right)=\left(\left(\mathcal{A}^{\dagger}\right)^{2} \mathcal{A}^{2} u, u\right)= \\
& \left(\mathcal{A}^{\dagger}\left(\mathcal{A} \mathcal{A}^{\dagger}-2 b\right) \mathcal{A} u, u\right)= \\
& \left\|\mathcal{A}^{\dagger} \mathcal{A} u\right\|^{2}-2(b \mathcal{A} u, \mathcal{A} u) \leq \\
& \left\|\mathcal{A}^{\dagger} \mathcal{A} u\right\|^{2}+2\|b\|_{\infty}\|\mathcal{A} u\|^{2} \leq \\
& \left\|\mathcal{A}^{\dagger} \mathcal{A} u\right\|^{2}+2\|b\|_{\infty}\left\|\mathcal{A}^{\dagger} \mathcal{A} u\right\|\|u\| .
\end{aligned}
$$

When $A^{(1)} \in C^{1}$, we approximate $A^{(1)}$ by $C^{\infty}$. potentials w.r.t. $C^{1}$-norm on some neighborhood of $\operatorname{supp} u$, then we get the above inequality again. Then, we have (17) by using (16). The case $X=Y=\mathcal{A}^{\dagger}$ can be treated similarly.

Proof of Proposition 3.5 First, by assumption (vi), we have

$$
\begin{aligned}
g^{-1} & =I+\hat{g}, \quad \max \left|\hat{g}_{m n}\right|=O\left(r^{2}\right), \\
G & =1+O\left(r^{2}\right), \quad|D G|=O(r),
\end{aligned}
$$

as $r \rightarrow 0$.
Define a unitary operator $U$ from $L^{2}\left(\mathbb{R}^{2} ; \sqrt{G} d x^{1} d x^{2}\right)$ to $L^{2}\left(\mathbb{R}^{2} ; d x^{1} d x^{2}\right)$ by

$$
U u=G^{1 / 4} u .
$$

Put $\tilde{\mathcal{L}_{k}}=U \mathcal{L}_{k} U^{-1}, \tilde{L}_{k, \min }=U L_{k, \min } U^{-1}$, etc. Then we have for $v \in C_{0}^{\infty}\left(\mathbb{R}^{2} \backslash\{0\}\right)$

$$
\tilde{L}_{k, \min } v=G^{-1 / 4}(D+A) \cdot \sqrt{G} g^{-1}(D+A) G^{-1 / 4} v \text {. }
$$

Thus we have

$$
\begin{aligned}
\tilde{L}_{k, \min }= & G^{-1 / 4}(D+A) \cdot G^{1 / 4} g^{-1}(D+A)+ \\
& G^{-1 / 4}(D+A) \cdot \sqrt{G} g^{-1}\left(D G^{-1 / 4}\right) .
\end{aligned}
$$

The second term of (19) is written as

$$
\begin{aligned}
& G^{-1 / 4}\left(D \cdot\left(\sqrt{G} g^{-1}\left(D G^{-1 / 4}\right)\right)\right)+ \\
& \left(D G^{-1 / 4}\right) \cdot G^{1 / 4} g^{-1}(D+A) .
\end{aligned}
$$

The first term of (20) is bounded, and the second is infinitesimally small w.r.t. $M_{k \text {,min }}$, by Lemma 3.6. The first term of (19) is written as

$$
\begin{aligned}
& (D+A) \cdot g^{-1}(D+A)+ \\
& G^{-1 / 4}\left(D G^{1 / 4}\right) \cdot g^{-1}(D+A) .
\end{aligned}
$$

The second term of (21) is also infinitesimally small w.r.t. $M_{k, \min }$, by Lemma 3.6. The first term of (21) is written as

$$
M_{k, \min }+(D+A) \cdot \hat{g}(D+A) .
$$

The second term of this expression is written as

$$
\begin{aligned}
& \sum_{m, n=1,2}\left(D_{m} \hat{g}_{m n}\right)\left(D_{n}+A_{n}\right)+ \\
& \sum_{m, n=1,2} \hat{g}_{m n}\left(D_{m}+A_{m}\right)\left(D_{n}+A_{n}\right) .
\end{aligned}
$$

The first sum of (22) is infinitesimally small w.r.t. $M_{k, \min }$. If we take $\epsilon_{k}$ sufficiently small, the second sum is $M_{k, \text { min- }}$-bounded with relative bound less than 1, by Lemma 3.6. Now we can apply Lemma 3.4, and conclude that $D\left(\tilde{L}_{k, \min }\right)=D\left(M_{k, \min }\right)=D\left(M_{k, \min }^{(0)}\right)$, and $n_{ \pm}\left(L_{k, \min }\right)=n_{ \pm}\left(\tilde{L}_{k, \min }\right)=n_{ \pm}\left(M_{k, \min }\right)=$ $n_{ \pm}\left(M_{k, \text { min }}^{(0)}\right)$. Moreover, one can show that multiplication by $G^{1 / 4}$ is a bijective continuous map on $D\left(M_{k, \min }^{(0)}\right)$. Thus we have

$$
\begin{aligned}
& D\left(L_{k, \min }\right)=U^{-1} D\left(\tilde{L}_{k, \min }\right)= \\
& G^{-1 / 4} D\left(M_{k, \min }^{(0)}\right)=D\left(M_{k, \min }^{(0)}\right)
\end{aligned}
$$

And then we can prove $\mathcal{L}_{k} f_{k}^{m} \in L^{2}\left(\mathbb{R}^{2} ; d \mu_{k}\right)$ by the Leibniz formula and (18), and thus $\left\{\left[f_{k}^{m}\right]\right\}_{m}$ form a basis of $\mathcal{D}_{k}$. 
In a similar way as in (13), we have

$$
\begin{aligned}
{[u, v]_{\mathcal{D}_{k}}=} & \lim _{\epsilon \rightarrow 0} \int_{r=\epsilon}\left(v n \cdot \sqrt{G} g^{-1} \overline{(\nabla+i A) u}-\right. \\
& \left.-\bar{u} n \cdot \sqrt{G} g^{-1}(\nabla+i A) v\right) r \mathrm{~d} \theta
\end{aligned}
$$

Since $\sqrt{G} g^{-1}=I+O\left(r^{2}\right)$, we can replace $\sqrt{G} g^{-1}$ by $I$ in the calculation of $\left[f_{k}^{m}, f_{k}^{n}\right]_{\mathcal{D}_{k}}$, and we have $\left[f_{k}^{m}, f_{k}^{n}\right]_{\mathcal{D}_{k}}=\left[f_{k}^{m}, f_{k}^{n}\right]_{\mathcal{E}_{k}}$. Thus we have the conclusion.

\section{Proof of main theorems}

Proof of Theorem 1.1 Since $H_{\text {min }}$ is semibounded, we have $n_{+}\left(H_{\min }\right)=n_{-}\left(H_{\min }\right)=$ $\operatorname{dim} \mathcal{D} / 2$. By Lemma 3.1 and Proposition 3.5, we have for $K<\infty$

$$
\operatorname{dim} \mathcal{D}=\sum_{k=1}^{K} \operatorname{dim} \mathcal{D}_{k}=4 K_{1}+2 K_{2},
$$

and for $K=\infty$

$$
\operatorname{dim} \mathcal{D} \geq \sum_{k=1}^{\infty} \operatorname{dim} \mathcal{D}_{k}=\infty
$$

Thus we have the conclusion.

Proof of Theorem 1.2 By Lemma 3.1 and Proposition 3.5 , we have for $u, v \in D\left(H_{\max }\right)$

$$
[u, v]_{\mathcal{D}}=4 \pi \Phi(u)^{*}\left(\begin{array}{cc}
O & -D \\
D & O
\end{array}\right) \Phi(v),
$$

where $\Phi(u)^{*}$ is the row-vector ${ }^{t} \overline{\Phi(u)}$ and $D$ is the matrix given by (6). Let $X={ }^{t}\left(X_{1}, X_{2}\right)$ be the matrix satisfying (7). Then we have

$$
X^{*}\left(\begin{array}{cc}
O & -D \\
D & O
\end{array}\right) X=O
$$

which implies $V \subset V^{[\perp]}$ for $V=\operatorname{Ran} X$. Moreover, if $\operatorname{rank} X=2 K_{1}+K_{2}$, we have

$\operatorname{dim} V^{[\perp]}=4 K_{1}+2 K_{2}-\operatorname{dim} V=2 K_{1}+K_{2}=\operatorname{dim} V$.

Thus we have (11), and therefore $H_{X}$ is self-adjoint. Conversely, for a given self-adjoint extension $H$ of $H_{\text {min }}$, we can construct a $\left(4 K_{1}+2 K_{2}\right) \times\left(2 K_{1}+K_{2}\right)$ matrix $X$ by arranging the coefficients of an arbitrary basis of $V=P D(H)$ with respect to the basis $\left\{\left[\psi_{k} f_{k}^{j}\right]\right\}$.

\section{$5 \quad$ Infinite singularities}

Let us consider the case $K=\infty$, and extend Theorem 1.2. Even in this case, for $u \in D\left(H_{\max }\right)$ and for each $k$, we can define the asymptotic coefficients $c_{j}^{k}$ at $\gamma_{k}$. However, the sequence $\Phi_{j}(u)$ is an infinite sequence. We shall find appropriate assumptions which make these infinite sequences square summable.

In the sequel, $U_{k}, \beta_{k}, g_{m n}$ are those introduced in section 1 . However, we may replace $\psi_{k}$ defined by (3) more appropriate one satisfying (4), if such one exists. For simplicity, we assume $V=0$.

(U) (i) There exists $\epsilon_{0}>0$, independent of $k$, such that $U_{k}=\left\{r<\epsilon_{0}\right\}$ for every $k$.

(ii) There exist $\beta_{-}, \beta_{+}$such that $0<\beta_{-} \leq$ $\beta_{k} \leq \beta_{+}<1$ or $\beta_{k}=0$, for every $k$.

(iii) There exists $C_{1}>0$ independent of $k$ such that $g_{m n}$ satisfies (2) and

$$
\left|\partial_{i} \partial_{j} g_{m n}\right| \leq C_{1}
$$

in $U_{k}$, for every $i, j, m, n=1,2$.

(iv) There exists $C_{2}>0$ independent of $k$, and phase functions $\psi_{k} \in C^{\infty}\left(U_{k} \backslash\{0\}\right)$ satisfying $\left|\psi_{k}\right|=1$, (4) and

$$
\left|\partial_{j} A_{m}^{(1)}\right| \leq C_{2}
$$

in $U_{k}$, for $j, m=1,2$.

Thus we assume some homogeneity for $g, A^{(0)}$, and $A^{(1)}$. Since the open sets $\left\{U_{k}\right\}_{k=1}^{\infty}$ are required to be disjoint, assumption (i) says the points of $\Gamma$ are uniformly separated in some sense. Assumption (ii) seems a little strange, but we need this assumption if we want to make the boundary value $\Phi(u)$ square summable. $^{7}$ Assumption (iii) binds the curvature of $M$, and (iv) the intensity of the magnetic field. In [12], the author considers a similar assumption when $M$ is the flat Euclidean plane and $d A^{(1)}$ is a constant magnetic field.

In the sequel, we use the notation

$$
\mathbb{C}^{\infty}=l^{2}=\left\{\left.\left(c_{j}\right)_{j=1}^{\infty}\left|\sum_{j=1}^{\infty}\right| c_{j}\right|^{2}<\infty\right\},
$$

and define its inner product by usual $l^{2}$-inner product. Let

$$
\mathcal{H}=\mathbb{C}^{K_{1}} \oplus \mathbb{C}^{K_{1}} \oplus \mathbb{C}^{K_{2}}
$$

Proposition 5.1 Assume (A), (AO), (A1), (SB), (U), $V=0$, and $K=\infty$. Then, the following linear map

\footnotetext{
${ }^{7}$ If we consider another type of characterization, assumption (ii) may be dropped.
} 


$$
\mathcal{D} \ni[u] \mapsto \Phi(u) \in \mathcal{H} \oplus \mathcal{H}
$$

is a well-defined homeomorphism. Moreover,

$$
\begin{aligned}
{[u, v]_{\mathcal{D}} } & =4 \pi(\Phi(u), \tilde{D} \Phi(v)), \\
\tilde{D} & =\left(\begin{array}{cc}
O & -D \\
D & O
\end{array}\right),
\end{aligned}
$$

where $\mathcal{D}$ is a bounded operator on $\mathcal{H}$ defined by (6).

Once this proposition is established, our theorem can be proved similarly as in the proof of Theorem 1.2. So we omit the proof.

Theorem 5.2 Assume the same conditions as in Proposition 5.1. Then, the statements of Theorem 1.2 hold with the following changes: $X_{1}, X_{2}$ are bounded operators on $\mathcal{H}$, and condition (7) is replaced by the condition

$$
\operatorname{Ran} X=\operatorname{Ker} X^{*} \tilde{D}
$$

where $\tilde{D}$ is the bounded operator on $\mathcal{H} \oplus \mathcal{H}$ defined in Proposition 5.1.

We conclude this paper by proving Proposition 5.1.

Proof of Proposition 5.1. We divide the proof into two steps.

Step 1. The map

$$
\mathcal{D} \ni[f] \mapsto \bigoplus_{k=1}^{\infty} T_{k}[f] \in \bigoplus_{k=1}^{\infty} \mathcal{D}_{k}
$$

is continuous, bijective and its inverse is also continuous.

Proof. By our assumption (U) and the calculation in section 3 , we can prove there exists $C>0$ independent of $k$ such that

$$
\left\|\psi_{k}^{-1} \chi_{k} f\right\|_{L_{k, \max }}^{2} \leq C \int_{U_{k}}\left(|\mathcal{L} f|^{2}+|f|^{2}\right) d \mu_{k} .
$$

Summing up these equalities with respect to $k$, we conclude the map

$$
D\left(H_{\max }\right) \ni f \mapsto \bigoplus_{k=1}^{\infty} \psi_{k}^{-1} \chi_{k} f \in \bigoplus_{k=1}^{\infty} D\left(L_{k, \max }\right)
$$

is continuous. Then the well-definedness of the map (23) can be proved similarly as in section 3. Since $\mathcal{D}$ is identified with the closed subspace $D\left(H_{\min }\right)^{\perp}$ of $D\left(H_{\max }\right)$ and the projection from $D\left(L_{k, \max }\right)$ to $\mathcal{D}_{k}$ is continuous, we conclude the map (23) is continuous. Moreover, we can prove the inverse map is also well-defined and continuous, so we have the conclusion.

Step 2. There exists $C>1$ independent of $k$ such that

$$
C^{-1}\left|c^{k}\right| \leq\|[u]\|_{\mathcal{D}_{k}} \leq C\left|c^{k}\right|
$$

for every $[u] \in \mathcal{D}_{k}$, where $c^{k}=\left(c_{1}^{k}, c_{2}^{k}, c_{4}^{k}, c_{5}^{k}\right)$ for $0<\beta_{k}<1, c^{k}=\left(c_{3}^{k}, c_{6}^{k}\right)$ for $\beta_{k}=0$, and $c_{j}^{k}$ are asymptotic coefficients of $u$ defined in section 1 .

Proof. We only consider the case $0<\beta_{k}<1$. Consider the following formula for $c_{1}^{k}$

$$
c_{1}^{k}=\frac{1}{4 \pi\left(1-\beta_{k}\right)}\left[f_{k}^{4}, u\right]_{\mathcal{D}_{k}},
$$

which can be verified by substituting all the basis functions into $u$. By choosing the representative $u \in D\left(L_{k, \min }\right)^{\perp}\left(\right.$ so $\left.\|u\|_{L_{k, \max }}=\|[u]\|_{\mathcal{D}_{k}}\right)$ and using the Schwarz inequality, we have

$$
\left|c_{1}^{k}\right| \leq \frac{1}{2 \pi\left(1-\beta_{k}\right)}\left\|f_{k}^{4}\right\|_{L_{k, \max }}\|[u]\|_{\mathcal{D}_{k}}
$$

The fraction is bounded uniformly w.r.t. $k$, by our assumption (ii) of (U). Moreover, we can prove $\left\|f_{k}^{j}\right\|_{L_{k, \max }}$ is also uniformly bounded, by (U) and the calculations in section 3 (first decompose $\mathcal{L}_{k}$ as in section 3 , and estimate all terms). Thus we have

$$
\left|c_{j}^{k}\right| \leq C\|[u]\| \|_{\mathcal{D}_{k}} .
$$

for $j=1$. The case $j=2,4,5$ can be treated similarly.

Conversely,

$$
\sum_{j=1,2,4,5}\left\|c_{j}^{k}\left[f_{k}^{j}\right]\right\|_{\mathcal{D}_{k}} \leq\left|c^{k}\right|\left(\sum_{j=1,2,4,5}\left\|f_{k}^{j}\right\|_{L_{k, \max }}^{2}\right)^{1 / 2}
$$

and the sum in the right hand side is uniformly bounded. Thus the conclusion holds.

By Step 1 and 2, we have proved the map (23) is well-defined and homeomorphism. Equation (24) is confirmed by substituting each $f_{k}^{j}$ as $u$ or $v$.

\section{Acknowledgement}

This work was partially supported by Doppler Institute for mathematical physics and applied mathematics, KIT Faculty Research Abroad Fellowship Program, and JSPS grant Wakate 20740093.

\section{References}

[1] Adami, R., Teta, A.: On the Aharonov-Bohm Hamiltonian, Lett. Math. Phys. 43 (1998), $43-54$.

[2] Aharonov, Y., Bohm, D.: Significance of electromagnetic potentials in the quantum theory, Phys. Rev. 115 (1959), 485-491. 
[3] Albeverio, S., Gesztesy, F., Høegh-Krohn, R., Holden, H.: Solvable models in quantum mechanics. Texts and Monographs in Physics., Springer-Verlag, New York, 1988.

[4] Bulla, W., Gesztesy, F.: Deficiency indices and singular boundary conditions in quantum mechanics, J. Math. Phys. 26 No. 10 (1985), 2520-2528.

[5] Correa, F., Falomir, H., Jakubsky, V., Plyushchay, M. S.: Hidden superconformal symmetry of spinless Aharonov-Bohm system preprint, URL: http://arxiv.org/abs/0906.4055

[6] Correa, F., Falomir, H., Jakubsky, V., Plyushchay, M. S.: Supersymmetries of the spin$1 / 2$ particle in the field of magnetic vortex, and anyons, preprint,

URL: http://arxiv.org/abs/1003.1434

[7] Dabrowski, L., Stovíček, P.: Aharonov-Bohm effect with $\delta$-type interaction, J. Math. Phys. 39, No. 1 (1998), 47-62.

[8] Exner, P., Sttovíček, P., Vytřas, P.: Generalized boundary conditions for the AharonovBohm effect combined with a homogeneous magnetic field, J. Math. Phys. 43, No. 5 (2002), 2151-2 167 .
[9] Iwai, T., Yabu, Y.: Aharonov-Bohm quantum systems on a punctured 2-torus, J. Phys. A: Math. Gen. 39 (2006) 739-777.

[10] Kato, T.: Perturbation theory for linear operators. Springer, 1966.

[11] Lisovyy, O.: Aharonov-Bohm effect on the Poincaré disk, J. Math. Phys. 48 (2007), no. 5, 052112 .

[12] Mine, T.: The Aharonov-Bohm solenoids in a constant magnetic field. Ann. Henri Poincaré 6 (2005), no. 1, 125-154.

[13] Reed, M., Simon, B.: Methods of modern mathematical physics. II. Fourier analysis, selfadjointness, Academic Press, New York-London, 1975.

[14] Shubin, M.: Essential Self-adjointness for Semibounded Magnetic Schrödinger Operators on Non-compact Manifolds, J. Funct. Anal. 186 (2001), 92-116.

Dr. Takuya Mine

E-mail: mine@kit.ac.jp

Kyoto Institute of Technology

Matsugasaki, Sakyo-ku

Kyoto 606-8585, Japan 